\title{
Employing the thiol-ene click reaction via metal-organic frameworks for integrated sonodynamic-starvation therapy as an oncology treatment
}

\author{
Sen Jiang ${ }^{1}$, Qijia $\mathrm{He}^{3}$, Chengcheng $\mathrm{Li}^{1}$, Kun Dang ${ }^{1}$, Ling $\mathrm{Ye}^{2}$, Weiwei Zhang ${ }^{3^{*}}$ and Yang Tian ${ }^{{ }^{*}}$
}

\begin{abstract}
Hypoxia in the tumor microenvironment (TME) greatly limits the tumor-killing therapeutic efficacy of sonodynamic therapy (SDT); this phenomenon is further exacerbated by increased glutathione (GSH) levels in cancer cells. Simultaneously, cancer starvation therapy is increasingly recognized nowadays as a promising clinical translation, but the efficacy of glucose oxidase (GOx)-based starvation therapy is also limited by the lack of oxygen in the tumor. Glyceraldehyde-3-phosphate dehydrogenase (GAPDH) is a key glycolytic enzyme and can therefore be a target for starvation therapy in the absence of oxygen engagement. Here, we proposed thiol-ene click reactions based on a two-dimensional metal-organic framework (MOF) modification for tumor treatments to enable the combination of SDT and starvation therapy. Experimental studies demonstrated that the prepared material could consume GSH and GAPDH free from oxygen in TME, which benefited from the thiol-ene click reactions between the MOFs and thiol substances in cancer cells. Further experiments in vitro and in vivo indicated the prepared MOF materials could kill cancer cells efficiently. This study is expected to create a promising avenue for thiol-ene click reactions in SDT and starvation therapy for cancer.
\end{abstract}

Keywords: metal-organic framework, sonodynamic therapy, starvation therapy, thiol-ene click reactions

\section{INTRODUCTION}

Sonodynamic therapy (SDT) is an attractive therapeutic approach for killing malignant tumors due to its noninvasiveness, site-confined irradiation, and high tissue penetration depth [1-4]. The antitumor effect of SDT is highly dependent on the reactive oxygen species (ROS) caused by acoustic sensitizers and low-intensity ultrasound (US). During the SDT process, tumor cells typically produce high levels of endogenous antioxidant anti-glutathione (GSH) [5-9], attenuating the intracellular ROS level and hindering the efficiency of SDT. To address this issue, researchers often use nanomaterials, such as $\mathrm{MnWO}_{X}$ [10], $\mathrm{MnO}_{2}$ [11,12], and $\mathrm{Au}[13]$ to achieve GSH depletion [7,14]. The consumption of GSH by these nanomaterials is highly dependent on the levels of intratumoral $\mathrm{H}_{2} \mathrm{O}_{2}$ and $\mathrm{O}_{2}$. However, the partial pressure of $\mathrm{O}_{2}$ inside a tumor is less than $5 \mathrm{mmHg}$, and the concentration of $\mathrm{H}_{2} \mathrm{O}_{2}$ is in the range of $10-50 \mu \mathrm{mol} \mathrm{L}{ }^{-1}$ [15-17]. These tumor microenvironments (TME) are insuffi- cient for nanomaterials to exert the effective and complete stifling of tumor growth and metastasis, resulting in the weakened efficacy of SDT $[4,6,18]$.

Cancer cells consume glucose through the glycolytic pathway to acquire energy for their growth, a phenomenon known as the "Warburg effect" [19-21]. The rapid proliferation of cancer cells requires an abundant source of nutrients and an adequate energy supply. If cancer cells are unable to obtain a sufficient supply of glucose and other nutrients to maintain their rapid growth, they may die of starvation, which is known as cancer starvation therapy [22-24]. Current starvation therapy is usually dependent on loading glucose oxidase (GOx) into tumors, which can consume glucose and turn it into gluconic acid [25-28]. However, $\mathrm{O}_{2}$ is essential for this process, so the efficacy of GOxbased starvation therapy is also severely limited by the tumor's hypoxic environment. Glyceraldehyde-3-phosphoglyceraldehyde dehydrogenase (GAPDH) is a crucial enzyme responsible for promoting the rate of glycolytic processing [29-31]. Therefore, reducing the glycolytic activity of GAPDH in TME would help cut off the energy source allowing cancer growth, thus improving starvation therapy without $\mathrm{O}_{2}$ participation.

Thiol-ene click reactions are simple and highly efficient reactions between thiols and carbon-carbon double bonds or carbon-carbon triple bonds that are mediated by free radicals [32-34]. Importantly, the reactions do not require the participation of oxygen to consume the thiol substances rapidly [3539]; this gives it huge potential for depleting the GSH and GAPDH molecules in TME [40], both of which include substantial quantities of thiols. Therefore, the application of the thiol-ene click reaction can overcome the current shortcomings of SDT, which is limited by hypoxia, and cut off the energy supply pathway used by cancer cells to achieve starvation therapy. Unfortunately, thiol-ene click reaction promoting SDT and cancer starvation therapy has not yet been reported as far as we know.

Here, we introduce the thiol-ene click reaction into the treatment of cancer to improve SDT and starvation therapies for cancer. We chose the porphyrin-based two-dimensional (2D) metal-organic frameworks (MOFs) as the ultrasonic sensitizer, which can generate free radicals under US due to the existence of porphyrin molecules [41]. Then, 5-norbornene-2-carbaldehyde (NC) molecules, which can rapidly react with a sulfhydryl group $[32,42]$, were linked onto the $2 \mathrm{D}$ Gd-TCPP $($ TCPP $=4$, $4^{\prime}, 4^{\prime \prime}, 4^{\prime \prime \prime}$-porphyrin-5,10,15,20-tetrayltetrabenzoic acid) nanosh-

\footnotetext{
${ }^{1}$ Department of Chemistry, Analytical Instrumentation Center, Capital Normal University, Beijing 10048, China

${ }^{2}$ School of Pharmaceutical Sciences, Capital Medical University, Beijing 100069, China

${ }^{3}$ College of Life Sciences, Capital Normal University, Beijing 10048, China

* Corresponding authors (emails: tianyang@cnu.edu.cn (Tian Y); zhangww@cnu.edu.cn (Zhang W))
} 
eets via ligand exchange. A series of in vitro experiments confirmed that the synthesized product could trigger thiol-ene click reactions with GSH and GAPDH in cells under US, thus promoting SDT and starvation therapy (Scheme 1). A range of in vivo experiments further demonstrated that the product could kill cancer cells with high efficiency and high biosafety. In addition, the product delivered a high relaxation rate of $T_{1}$ and was able to undergo magnetic resonance imaging (MRI) in mice in vivo due to the involvement of the Gd element, meaning that the product is promising as an MRI diagnosis-guided SDT and starvation therapy with an anticancer effect.

\section{EXPERIMENTAL SECTION}

\section{Chemicals}

TCPP, $N, N$-dimethyl formamide (DMF), gadolinium(III) nitrate hydrate $\left(\mathrm{Gd}\left(\mathrm{NO}_{3}\right)_{3}\right)$, 5-fluoroanthranilic acid (FAA), NC, GSH, ethanol, and ether were purchased from Shanghai Aladdin Chemical Industry. All chemicals were of analytical reagent grade and used without further purification. Ultrapure water was obtained by filtration with a Pincheng system $(R=$ $18.25 \mathrm{M} \Omega \mathrm{cm}$ ).

\section{Synthesis of Gd-TCPP 2D nanosheets}

The 2D MOF Gd-TCPP nanosheets were prepared according to reference [43]. Briefly, $0.03 \mathrm{mmol}$ of TCPP (237.3 mg) was dissolved in $12 \mathrm{~mL}$ of DMF, and $0.01 \mathrm{mmol}$ of $\mathrm{Gd}\left(\mathrm{NO}_{3}\right)_{3}(34.4 \mathrm{mg})$ was dissolved in $1 \mathrm{~mL}$ of ultrapure water. The two solutions were then mixed in a Teflon reactor and placed in an oven at $65^{\circ} \mathrm{C}$ for $10 \mathrm{~h}$. The precipitate obtained was washed with ether several times.

\section{Synthesis of ligand NC-FAA}

After dissolving $0.12 \mathrm{~mol}$ of $\mathrm{NC}(14.64 \mathrm{mg})$ in $20 \mathrm{~mL}$ of ethanol in a three-necked flask, $0.1 \mathrm{~mol}$ of FAA $(15.5 \mathrm{mg})$ was added. The solution was heated to reflux at $55^{\circ} \mathrm{C}$ for $40 \mathrm{~h}$. The solvent was removed by rotary evaporation to give brown solid, which was then dissolved with dichloro-methane. The precipitate that could not dissolve was discarded, and the resulting solution underwent rotary evaporation to remove the solvent, giving brown solid NC-FAA.

\section{Synthesis of Gd-TCPP-NC}

In a Teflon reactor, $27.2 \mathrm{mg}$ of Gd-TCPP and $27.5 \mathrm{mg}$ of NCFAA were dispersed in $12 \mathrm{~mL}$ of ethanol and reacted without stirring at $60^{\circ} \mathrm{C}$ for $12 \mathrm{~h}$. The mixture was centrifuged $\left(8000 \mathrm{r} \mathrm{min}^{-1}\right)$ to collect the precipitate, which was washed several times with ethanol to obtain Gd-TCPP-NC.

\section{Capture of $\cdot \mathrm{OH}$ and alkyl radicals by electron spin-resonance (ESR)}

5,5-Dimethyl-1-pyrroline $N$-oxide (DMPO) in $10 \mu \mathrm{L}$ was mixed with $50 \mu \mathrm{L}$ aqueous solution of the material in $100 \mu \mathrm{g} \mathrm{mL}^{-1}$ and subjected to US irradiation $\left(1.2 \mathrm{MHz}, 1.5 \mathrm{~W} \mathrm{~cm}^{-2}, 4 \mathrm{~min}\right)$. The signals of $\cdot \mathrm{OH}$ and alkyl radicals were monitored by an ESR spectrometer.

Detection of GSH and glutathione oxidized (GSSG) in solution Several aliquots were prepared by mixing GSH $(100 \mu \mathrm{L}$, $\left.10 \mathrm{mmol} \mathrm{L}^{-1}\right)$ with Gd-TCPP-NC nanosheets $(100 \mu \mathrm{L}$, $29 \mathrm{mg} \mathrm{mL}^{-1}$ ) in $800 \mu \mathrm{L}$ of water, and the supernatant was extracted from respective aliquots at different time points for GSSG and GSH content test by the reductive glutathione assay kit (purchased from the Suzhou Comin Biotechnology Company).

\section{Measurement of relaxivity of MRI}

Inductively coupled plasma (ICP) analysis was performed on $10 \mathrm{~mL}$ aqueous solutions of Gd-TCPP MOF nanosheets and aqueous solutions of Gd-TCPP-NC MOF nanosheets. The solutions were then diluted to $\mathrm{Gd}$ concentrations of $0.01,0.02$, $0.04,0.08$, and $0.1 \mathrm{mmol} \mathrm{L}^{-1}$. The $T_{1}$ values of the solutions with different concentrations were obtained with a 0.5 T NIUMAGMesoMR23-060H-1 at $32^{\circ} \mathrm{C}$.

\section{MTT assay for cytotoxicity}

Normal human tubular epithelial cells (HKC) and human kidney cancer cells (A498) were inoculated on 96-well plates and

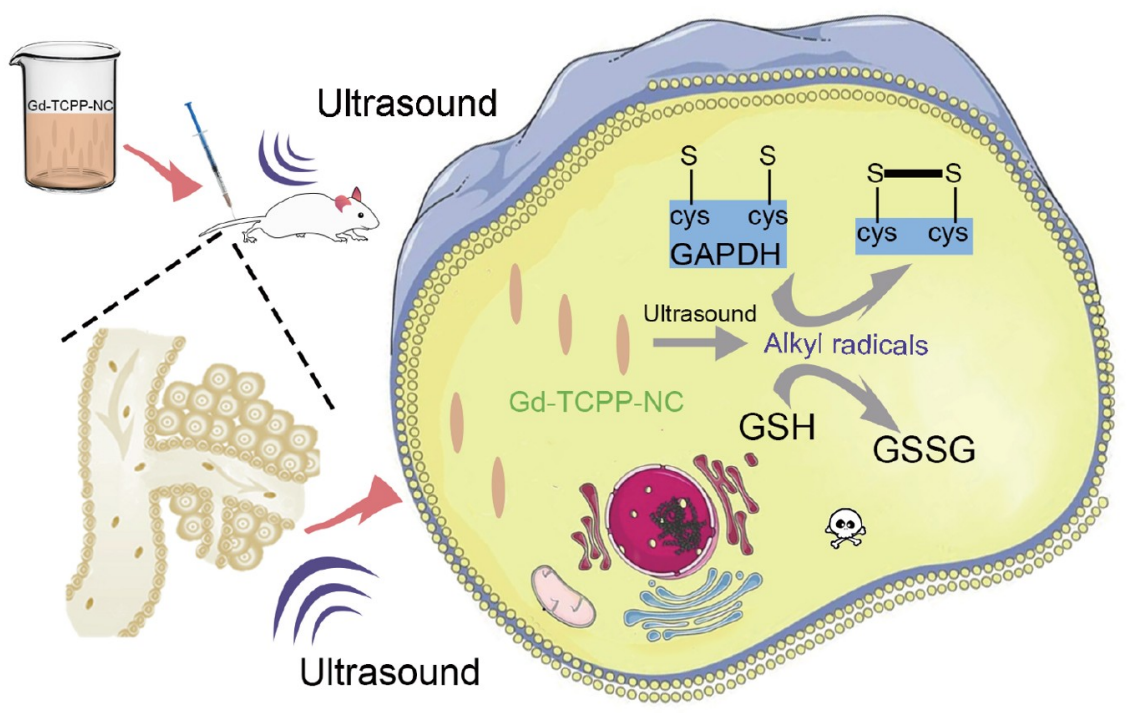

Scheme 1 Schematic diagram of the prepared 2D MOF of Gd-TCPP-NC used for SDT and starvation therapy of tumor via thiol-ene click reaction. 
incubated with Dulbecco's modified Eagle medium (DMEM, about $1 \times 10^{4}$ cells per well) for $24 \mathrm{~h}$. Then, these cells were incubated with different concentrations of $0-100 \mu \mathrm{g} \mathrm{mL}^{-1}$ of GdTCPP or Gd-TCPP-NC nanosheets for another $24 \mathrm{~h}$. 3-[4,5Dimethylthiazole-2-yl]-2,5-diphenyltetrazolium bromide (MTT $\left.5 \mathrm{~g} \mathrm{~L}^{-1}, 10 \mu \mathrm{L}\right)$ and dimethyl sulfoxide $(100 \mu \mathrm{L})$ were added and shaken for $10 \mathrm{~min}$, and the cells were incubated in the dark for $30 \mathrm{~min}$. Each well was measured at $570 \mathrm{~nm}$ with an enzyme marker. Cell viability was calculated by comparing the absorbance of treated cells with that of the control. HKC cells and A498 cells were also treated with US for $5 \mathrm{~min}$, and then the absorbance at $570 \mathrm{~nm}$ was measured for comparison of cell viability.

\section{Determination of GSH, GAPDH, and pyruvate in cells}

After incubation, HKC and A498 cells were collected from the culture dishes by treatment with trypsin and centrifugation, and the contents of GSH, GAPDH, and pyruvate in the cells were determined according to the GSH kit, GAPDH kit, and pyruvate kit, respectively.

\section{Treatment of tumor-bearing mice}

All animal experiments were performed according to the guidelines established by the Capital Normal University. The procedures for care and use of animals were approved by the Institutional Animal Care and Use Committee (IACUC) of Capital Normal University. Approximately $2 \times 10^{6}$ cells in phosphate buffer saline (PBS, $100 \mu \mathrm{L}$ ) were injected subcutaneously into the back of each group of six week-old male $\mathrm{BALB} / \mathrm{c}$ mice (Beijing Charles River Company, License number: SCXK 2016-0006). When the tumor volume reached the desired volume (about $1500 \mathrm{~mm}^{3}$ ), in vivo experiments were performed.

To assess the therapeutic effect of the material at the animal level, we randomly divided the mice with implanted A498 tumors into three groups ( $n=4$ each group): (i) PBS $\left(10 \mathrm{~mL} \mathrm{~kg}^{-1}\right)$; (ii) Gd-TCPP $\left(600 \mu \mathrm{g} \mathrm{mL}^{-1}, 10 \mathrm{~mL} \mathrm{~kg}{ }^{-1}\right)$; (iii) GdTCPP-NC with the same dose as Gd-TCPP. The mice were treated with US every day $(1.2 \mathrm{MHz}$ with a power density of $1.5 \mathrm{~W} \mathrm{~cm}^{-2}$ and a duty cycle of $50 \%$ for $5 \mathrm{~min}$ ), for a total of five days [44-47]. PBS, Gd-TCPP or Gd-TCPP-NC was injected via the tail vein of the mouse. The tumor size and weight were measured daily. The tumor size was estimated as follows: $V=a \times$ $b^{2} / 2$ ( $a$ : the longest tumor diameter; $b$ : the shortest tumor diameter).

MRI imaging of tumor-bearing mice and haematoxylin and eosin (H\&E) staining of organs

Homozygous mice injected with Gd-TCPP-NC $\left(600 \mu \mathrm{g} \mathrm{mL}^{-1}\right.$, $10 \mathrm{~mL} \mathrm{~kg}^{-1}$ mice) were anesthetized and imaged with the NIUMAG-MesoMR23-060H-1 at a predetermined time. On the $21^{\text {st }}$ day after receiving different US treatments, the mice were euthanized. The major organs (liver, spleen, kidney, heart, and lung) of mice were stained with $\mathrm{H} \& \mathrm{E}$ and observed under a microscope.

\section{RESULTS AND DISCUSSION}

Gd-TCPP MOF nanosheets were obtained in accordance with instructions from a reference (Figs S1-S6 in the Supplementary information) [43]. NC molecules were first modified with FAA to produce 2-(5-norbornene-2-carbaldehyde)-5-fluorobenzoic acid (NC-FAA), as shown in Fig. 1a (Figs S7 and S8). Then, the
Gd-TCPP nanosheets were linked with NC-FAA by replacing ligand water in Gd-TCPP with the NC molecules, resulting in the final product, which is denoted Gd-TCPP-NC (Fig. 1b, Figs S9 and S10).

Fourier-transform infrared spectroscopy (FTIR) was applied to confirm that the NC group was coordinated with Gd-TCPP. The FTIR spectrum of Gd-TCPP-NC in Fig. 1c (red curve) shows the characteristic peaks of $-\mathrm{C}-\mathrm{N}\left(1089.9 \mathrm{~cm}^{-1}\right)$ and $-\mathrm{N}-\mathrm{H}$ $\left(1606 \mathrm{~cm}^{-1}\right)$ in the TCPP ligands [48-50]. Compared with GdTCPP, a peak C-F bond at $1049.5 \mathrm{~cm}^{-1}$ appeared in the FTIR spectrum of Gd-TCPP-NC [51,52], which came from the NC molecules. This proves that the NC was combined with GdTCPP. This was further evidenced by the ultraviolet-visible (UVvis) absorption spectra (Fig. 1d) and fluorescence spectra (Fig. 1e). They demonstrate that the UV-vis absorption shifted from $421 \mathrm{~nm}$ of Gd-TCPP to $416 \mathrm{~nm}$ of Gd-TCPP-NC [53-55], and the fluorescence emission at $651 \mathrm{~nm}$ of Gd-TCPP shifted to $646 \mathrm{~nm}$ of Gd-TCPP-NC $[53,56]$. Both the blue-shifted UV-vis and fluorescence spectra were due to the F atoms in NC having a stronger electronegativity [54,55]. Transmission electron microscopy (TEM) and scanning electron microscopy (SEM) images (Fig. 1f, g) indicate the 2D nanosheet-like morphology of the prepared Gd-TCPP-NC with plane sizes in several hundred nanometers. Energy dispersive X-ray (EDX) spectroscopy in Fig. 1h demonstrates the Gd, F, N, C, and O elements in the GdTCPP-NC with a reasonable stoichiometric ratio.

ESR spectroscopy analysis was employed to investigate the Gd-TCPP-NC's generation of free radicals in the solution under US. We added the radical-trapping additive DMPO to the aqueous solution of Gd-TCPP-NC, which can combine with highly reactive $\cdot \mathrm{OH}$ and alkyl radicals to form stable paramagnetic adducts with ESR characteristics [5]. Fig. 2a shows that the aqueous solutions of Gd-TCPP and Gd-TCPP-NC could both produce $\cdot \mathrm{OH}$ under US. Fig. $2 \mathrm{~b}$ shows that the aqueous solution of Gd-TCPP could not produce alkyl radicals, while the aqueous solution of Gd-TCPP-NC induced alkyl radicals under US [57].

To confirm that the prepared Gd-TCPP-NC can be used for thiol-ene click reactions with GSH and GAPDH, we tested the depletion of GSH and the inhibition of GAPDH by Gd-TCPP and Gd-TCPP-NC in aqueous solutions under different conditions. As shown in Fig. 2c, in the absence of sonication, the GSH contents of aqueous solutions of both Gd-TCPP and Gd-TCPPNC remained above $90 \%$ for $2 \mathrm{~h}$. Under US, the content of GSH in Gd-TCPP solution dropped to 69\%, and the content of GSSG increased to approximately $31 \%$ in $2 \mathrm{~h}$ (Fig. $2 \mathrm{~d}$ ). In contrast, in the aqueous solution of Gd-TCPP-NC (Fig. 2d), the content of GSH declined to $20 \%$ under US, indicating Gd-TCPP-NC consumed much more GSH than Gd-TCPP. The results demonstrate the fact that Gd-TCPP-NC could react with GSH in a thiol-ene click reaction, while Gd-TCPP did not.

Similarly, the activity of GAPDH in Gd-TCPP and Gd-TCPPNC solutions remained above $94 \%$ for $2 \mathrm{~h}$ without US (Fig. 2e). Fig. $2 \mathrm{f}$ shows that the activity of GAPDH in Gd-TCPP solution was maintained at about $80 \%$ under US for $2 \mathrm{~h}$, while the activity of GAPDH in Gd-TCPP-NC solution was only about $40 \%$. This result indicates that Gd-TCPP-NC can rapidly inhibit the activity of GAPDH under US, which is expected to block the energy supply pathway of tumors and achieve effective tumor starvation therapy.

To give insight into whether Gd-TCPP-NC can effectively kill 


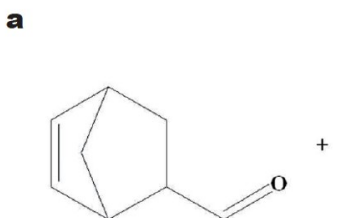

NC<smiles>Nc1ccc(F)cc1C(=O)O</smiles>

FAA

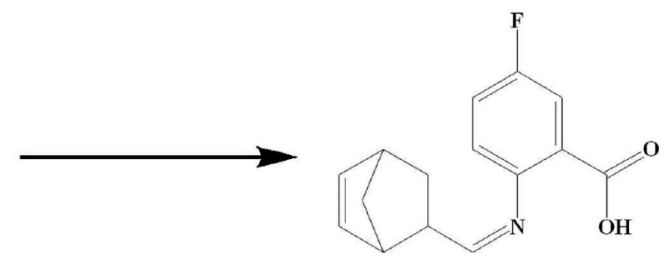

NC-FAA

b
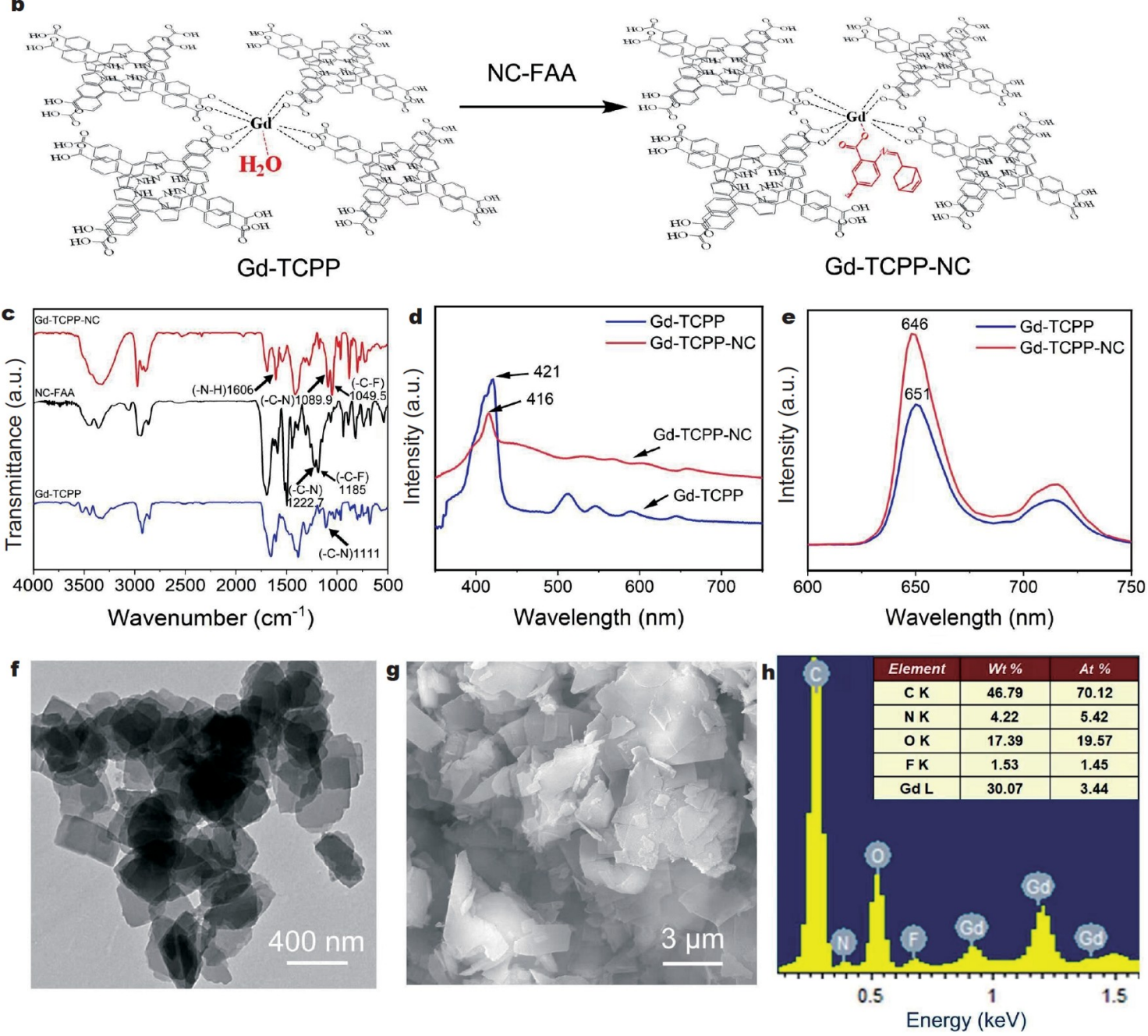

Figure 1 (a) Diagram showing the synthesis procedure of NC-FAA. (b) Diagram representing the fabrication of Gd-TCPP-NC nanosheets. (c) FTIR spectra of Gd-TCPP, NC-FAA, and Gd-TCPP-NC. (d) UV-vis absorption of Gd-TCPP and Gd-TCPP-NC. (e) Fluorescence signals of Gd-TCPP and Gd-TCPP-NC. TEM image (f), SEM image (g), and EDS spectrum (h) of Gd-TCPP-NC.

cancer cells through thiol-ene click reactions, we monitored the contents of GSH and GAPDH in A498 during the ultrasonic treatment of Gd-TCPP-NC with the control of Gd-TCPP. Here, GSH and GAPDH were analyzed by the kit method after incubation with different contents of Gd-TCPP-NC and Gd-TCPP. At a material concentration of 0 , the effect of ultrasound only on the intracellular GSH and GAPDH content was almost negligible, and the relative levels of both were above 95\% (Fig. S11). Fig. 3a shows that even at the low strength of $3.125 \mu \mathrm{g} \mathrm{m}^{-1}$ for Gd-TCPP-NC, the relative content of GSH in A498 declined by about $50 \%$, which is far greater than that of $5 \%$ for the Gd-TCPP treatment. Furthermore, all the tested GSH contents of GdTCPP-NC became much lower than that in Gd-TCPP at the same level. This demonstrates that the NC group in Gd-TCPP$\mathrm{NC}$ underwent thiol-ene click reactions and consumed large amounts of GSH under US [59,60].

Fig. $3 \mathrm{~b}$ shows that with an increase in the concentration of Gd-TCPP, the activity of GAPDH declined slightly under US treatment. The decline in the activity of GAPDH was up to $50 \%$, even at a low Gd-TCPP-NC concentration of $3.125 \mu \mathrm{g} \mathrm{mL} \mathrm{m}^{-1}$ (Fig. 3b). The reduction in GAPDH activity was attributed to the activation of the redox switch (cys152) of GAPDH by the alkyl radicals released from Gd-TCPP-NC [58]. At a concentration of $6.25 \mu \mathrm{g} \mathrm{mL}^{-1}$ of Gd-TCPP-NC or higher, we found that the activity of GAPDH increased instead (Fig. 3b). This was attributed to the alkyl radicals released from Gd-TCPP-NC at 

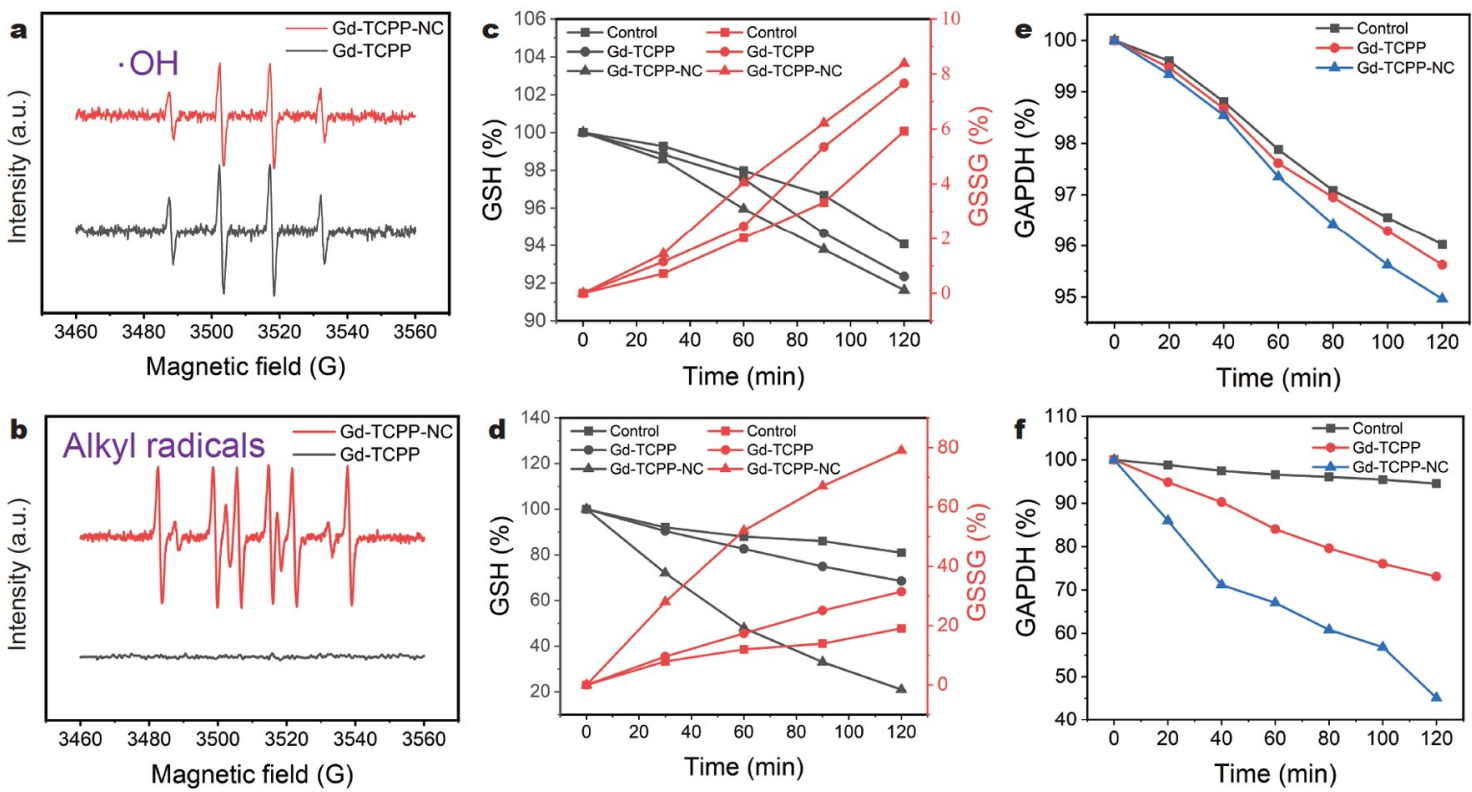

Figure 2 ESR spectra revealing (a) $\cdot \mathrm{OH}$ and (b) alkyl radicals of Gd-TCPP-NC and Gd-TCPP in water within US $\left(1.2 \mathrm{MHz}, 1.5 \mathrm{~W} \mathrm{~cm}^{-2}, 4 \mathrm{~min}, 50 \%\right.$ duty cycle). The contents of GSH and GSSG in the supernatant of Gd-TCPP and Gd-TCPP-NC at different time points (c) without US and (d) with US (1.2 MHz, $1.5 \mathrm{~W} \mathrm{~cm}^{-2}, 50 \%$ duty cycle). Inhibition of GAPDH in the Gd-TCPP and Gd-TCPP-NC solutions (e) without US and (f) with US (1.2 MHz, $1.5 \mathrm{~W} \mathrm{~cm}^{-2}, 50 \%$ duty cycle).
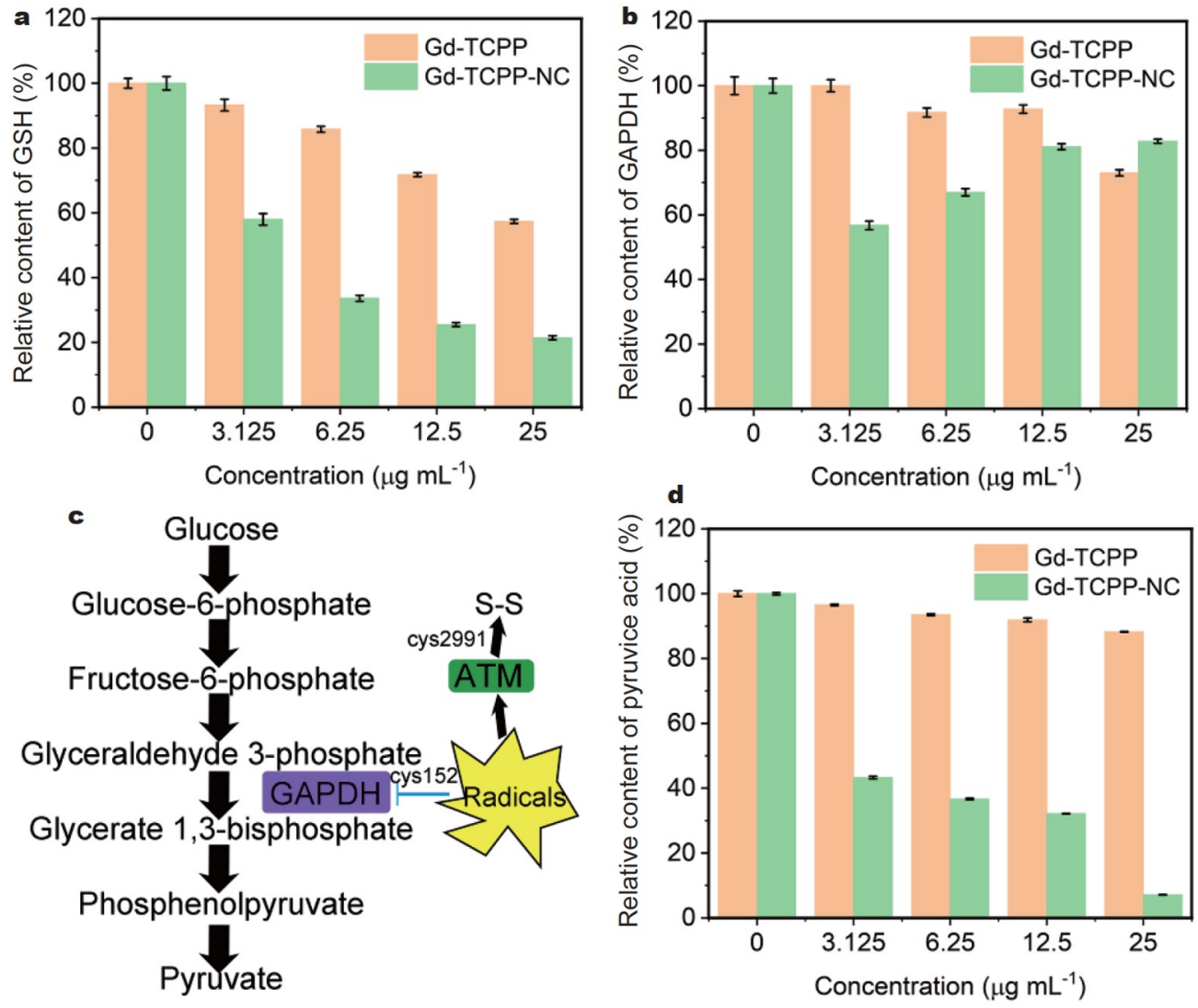

Figure 3 (a) Relative contents of GSH in A498 cells cultivated with Gd-TCPP and Gd-TCPP-NC under US normalized with that of US alone. (b) Relative contents of GAPDH in A498 cultivated with Gd-TCPP and Gd-TCPP-NC under US normalized with that of US alone. (c) Glycolysis process in carcinomas (modified from Ref. [58]). (d) Relative contents of pyruvic acid in A498 cultivated with Gd-TCPP and Gd-TCPP-NC under US normalized with that of US alone. 
high concentrations reacting with GAPDH and forming S-S in cys2991 [58], which further increased the GAPDH activity. Also, the formation of S-S in cys 2991 prevented glycolysis in cancer cells (Fig. 3c) [58]. This fact can be reinforced by the decrease of pyruvate (a product of GAPDH glycolysis) in corresponding cancer cells, as shown in Fig. $3 \mathrm{~d}$.

To verify the effectiveness of click reactions for killing cancer cells based on the prepared Gd-TCPP-NC consuming GSH and GAPDH in TME, HKC and A498 cells were incubated with the prepared materials. After 24-h incubation with Gd-TCPP and Gd-TCPP-NC without US, the survival rates of both HKC (Fig. 4a) and A498 (Fig. 4b) were above 85\%, even at the high material concentration of $50 \mu \mathrm{g} \mathrm{mL}^{-1}$ (Fig. $4 \mathrm{a}, \mathrm{b}$ ). This demonstrates that Gd-TCPP and Gd-TCPP-NC had low toxicity to HKC and A498 in the absence of US. In contrast, under US, the survival rate of HKC (Fig. 4c) decreased a little with the increase in the content of Gd-TCPP or Gd-TCPP-NC. The survival rate was kept at approximately $70 \%$ at the content of $25 \mu \mathrm{g} \mathrm{mL} \mathrm{L}^{-1}$ for Gd-TCPP or Gd-TCPP-NC. For A498 cancer cells (Fig. 4d), the survival rate decreased more clearly with the incubation of GdTCPP-NC under US. It was reduced to approximately $55 \%$ at a high concentration of $25 \mu \mathrm{g} \mathrm{mL}^{-1}$. This may be due to the different expression levels of GSH and GAPDH in normal cells and cancer cells. Moreover, the survival rate of A498 grown in GdTCPP-NC decreased more significantly than that in Gd-TCPP. Even at a low concentration of $3.125 \mu \mathrm{g} \mathrm{mL}^{-1}$, the survival rate of A498 grown in Gd-TCPP-NC was only 65\%, which is lower than that in $12.5 \mu \mathrm{g} \mathrm{mL} \mathrm{m}^{-1}$ of Gd-TCPP. These results suggest that Gd-TCPP-NC-produced alkyl radicals reacted with sulfhy-
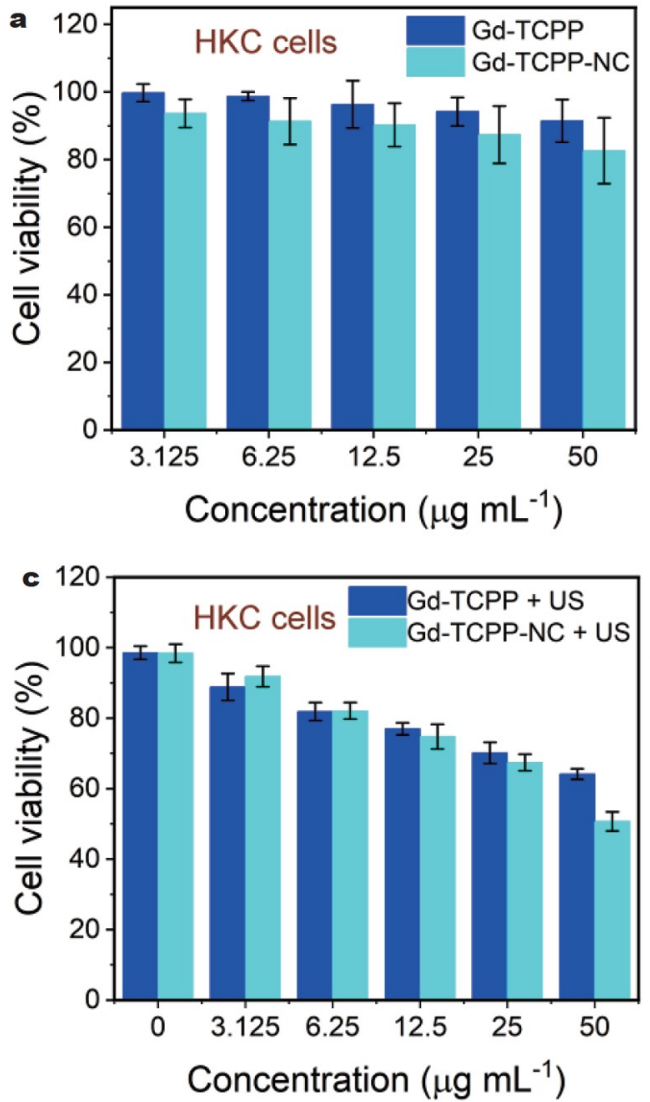

dryl units in the cells by the click reaction under US conditions (Figs S12-S14). Therefore, the killing of cancer cells is more effective by Gd-TCPP-NC than by Gd-TCPP under US.

To evaluate the ultrasonic therapeutic effects of the prepared Gd-TCPP-NC nanosheets in vivo, we injected A498 cancer cells subcutaneously into mice to obtain a tumor model. The tumorbearing mice were randomly divided into three groups $(n=4$ each group). The three groups of mice were injected once by PBS ( $\mathrm{pH} 7.5)$, Gd-TCPP solution $\left(600 \mu \mathrm{g} \mathrm{mL} \mathrm{mL}^{-1}\right)$, and Gd-TCPP-NC solution $\left(600 \mu \mathrm{g} \mathrm{mL}^{-1}\right)$ via tail vein, respectively, and the injection doses were all $10 \mathrm{~mL} \mathrm{~kg}^{-1}$. The three groups of mice were then irradiated with US daily ( $5 \mathrm{~min}, 1.2 \mathrm{MHz}, 1.5 \mathrm{~W} \mathrm{~cm}^{-2}, 50 \%$ duty cycle) for consecutive 5 days and executed after 21 days. As shown in Fig. 5a, b, as control, the tumors in the mice treated with PBS grew larger under US. However, the tumors volume in the mice treated with Gd-TCPP under US decreased gradually, and the tumor almost disappeared on the $21^{\text {st }}$ day. In contrast, the tumor in the mice treated with Gd-TCPP-NC under US disappeared on the $14^{\text {th }}$ day. These results indicate the GdTCPP-NC performed significant inhibition of tumor growth due to the thiol-ene click reactions between Gd-TCPP-NC and GAPDH/GSH in the tumor. After a one-month observation of the treated mice, there was no recurrence of tumor growth, and the mice showed a satisfactory growth status (Fig. S15). Further histological analysis by $H \& E$ staining showed no significant damage or inflammation in the tumor tissues treated with GdTCPP-NC and negligible side effects on the major organs (Fig. 5c); this further demonstrates the high biosafety of GdTCPP-NC.
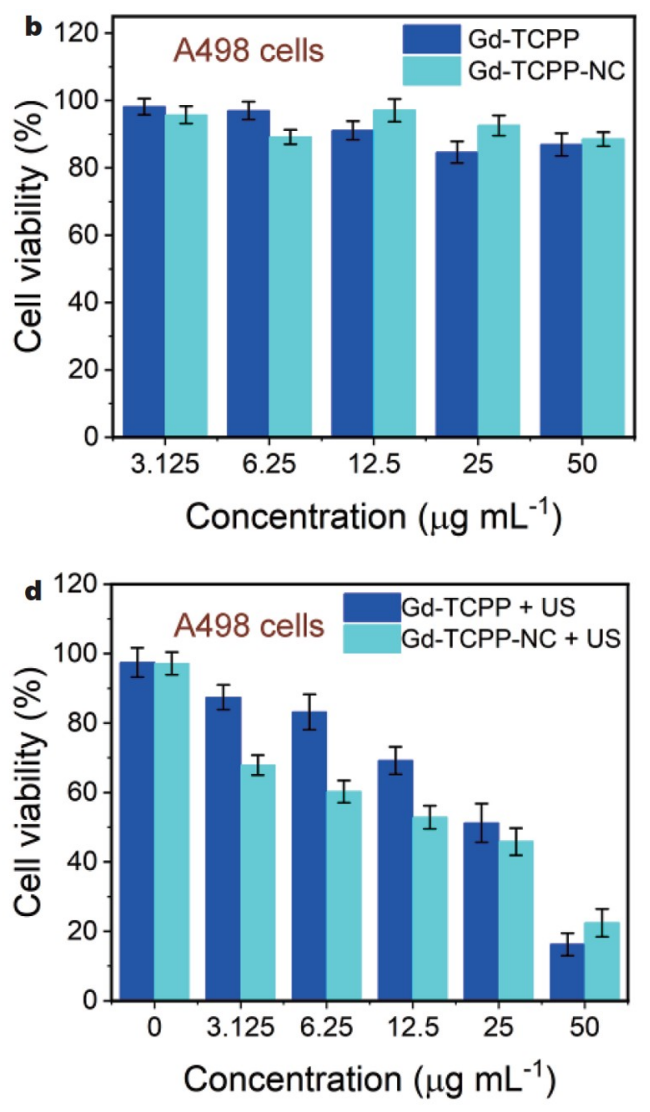

Figure 4 Cytotoxicity of Gd-TCPP and Gd-TCPP-NC on (a) HKC and (b) A498 without US. Cytotoxicity of Gd-TCPP and Gd-TCPP-NC on (c) HKC and (d) A498 under US. 


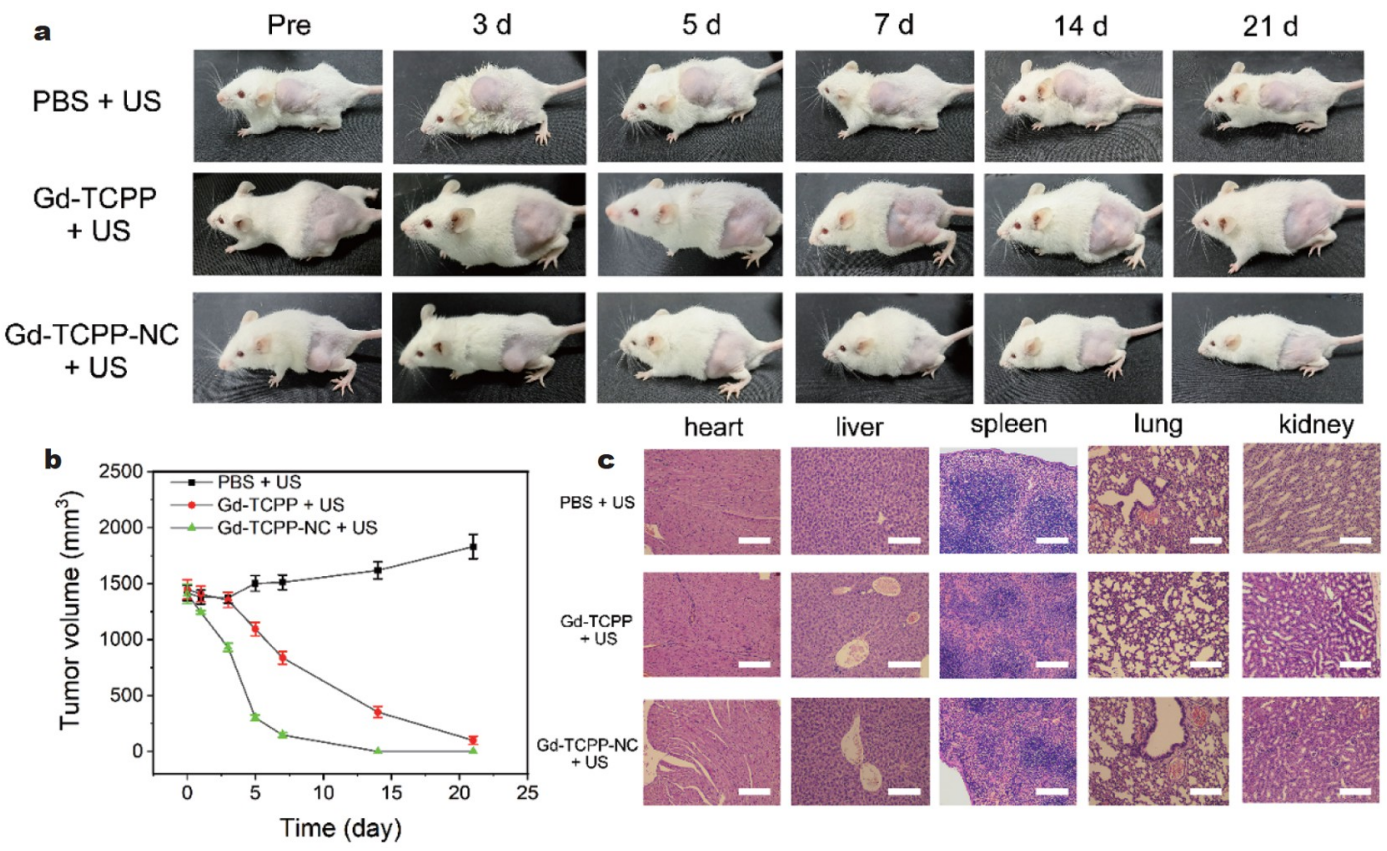

Figure 5 (a) Representative photos of A498 tumor-bearing mice after SDT treatments for 21 days. (b) Tumor growth curves of each group of mice. (c) H\&E staining of major organs (heart, liver, spleen, lung, and kidney) after receiving different US treatments on the 21 st day (scale bar: $50 \mu \mathrm{m}$ ).

a
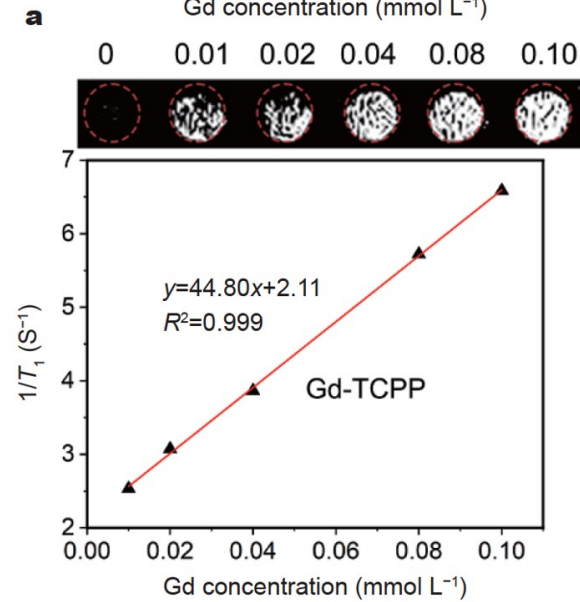

c

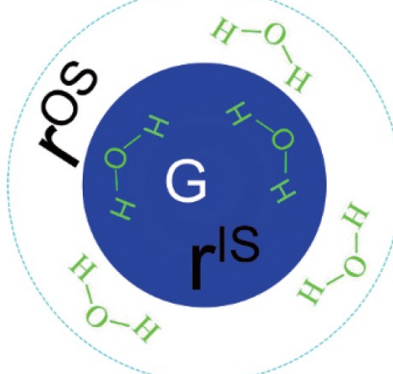

b

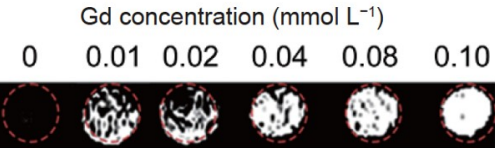

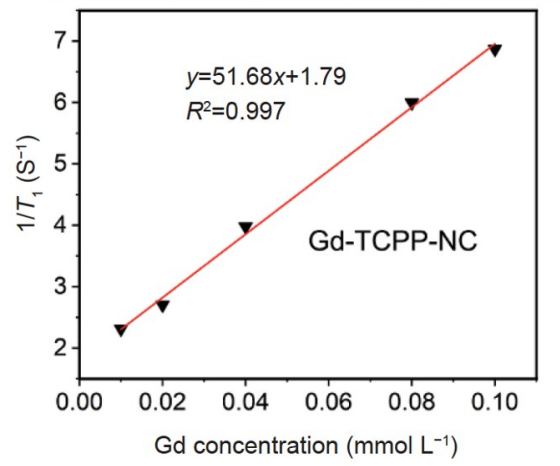

d $\mathrm{Oh}$
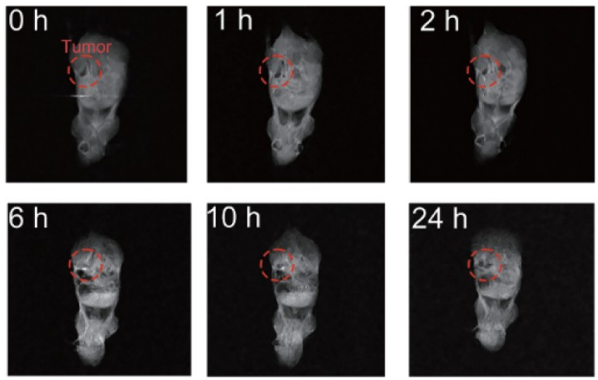

Figure 6 MRI imaging and relaxivity rates of (a) Gd-TCPP and (b) Gd-TCPP-NC in water. (c) Determinants of slackening rate $r^{\mathrm{IS}}$ and $r^{\mathrm{OS}}$. (d) $T_{1}$-weighted MRI images of tumor-bearing mice treated with Gd-TCPP-NC at different times.

In addition, due to the involvement of the Gd element, the prepared Gd-TCPP-NC nanosheets were also measured for potential MRI diagnosis. As a comparison, the Gd-TCPP presented a high $r_{1}$ relaxivity of $44.8 \mathrm{~S}^{-1}\left(\mathrm{mmol} \mathrm{L}^{-1}\right)^{-1}$ calculated by the computational slope from the fitted curve (Fig. 6a), which coincided with the value reported [43]. Interestingly, the $r_{1}$ relaxivity of the Gd-TCPP-NC increased to $51.7 \mathrm{~S}^{-1}$ $\left(\mathrm{mmol} \mathrm{L}^{-1}\right)^{-1}$ as seen from Fig. $6 \mathrm{~b}$, much higher than those of 
commercial contrast agents (Gd-DTPA: $\left.4.52 \mathrm{~S}^{-1}\left(\mathrm{mmol} \mathrm{L}^{-1}\right)^{-1}\right)$ and previously reported Gd-based materials (Table S1) [61,62]. The higher $r_{1}$ of Gd-TCPP-NC should be related to the innersphere water relaxivity $\left(r^{\mathrm{IS}}\right)$ and outer-sphere water relaxivity $\left(r^{\mathrm{OS}}\right)$, contributing to the restitution ratios per the SolomonBloembergen-Morgan (SBM) theory (Fig. 6c) [53,63]. For the $r^{\text {IS }}$, the rotational correlation time $\left(\tau_{\mathrm{R}}\right)$ of Gd-TCPP-NC was longer than that of Gd-TCPP due to the larger molecular weight, inducing stronger $r^{\mathrm{IS}}$ of Gd-TCPP-NC. For the $r^{\mathrm{OS}}$, diffusion correlation time $\left(\tau_{\mathrm{D}}\right)$ of the extraneous water around Gd-TCPPNC was longer than that around Gd-TCPP due to the higher hydrophobicity of Gd-TCPP-NC [64-66]. Therefore, the enhanced $r^{\mathrm{IS}}$ and $r^{\mathrm{OS}}$ of the Gd-TCPP-NC increased its $r_{1}$ relaxivity.

To confirm the potential of Gd-TCPP-NC for in vivo MRI, we injected $300 \mu \mathrm{L}$ of Gd-TCPP-NC solution $\left(600 \mu \mathrm{g} \mathrm{mL}^{-1}\right.$, $10 \mathrm{~mL} \mathrm{~kg}^{-1}$ mice dose) into an A498 tumor-bearing mouse via tail vein, which was then monitored in MRI. Fig. $6 \mathrm{~d}$ displays that during the period from 0 to $6 \mathrm{~h}$, the $T_{1}$ imaging of the tumor site gradually became brighter and clearer, indicating that GdTCPP-NC entered the tumor tissue due to its tumor-targeting ability. At $6 \mathrm{~h}$, the tumor site of the mouse had the strongest $T_{1^{-}}$ weighted MRI signal. The tumor signal gradually weakened during $10-24 \mathrm{~h}$, indicating that the Gd-TCPP-NC was gradually metabolized by the mouse, and signaling good biosafety of the material. The results suggest the prepared Gd-TCPP-NC had favorable potential as a $T_{1}$-weighted MRI contrast agent.

\section{CONCLUSIONS}

In summary, we prepared 2D MOF Gd-TCPP nanosheets coordinated with NC via ligand exchange. In vitro experiments showed that Gd-TCPP-NC can quickly exhaust GSH and suppress the glycolytic activity of GAPDH by undergoing a thiolene click reaction without the participation of oxygen, leading to a fall in the GSH level and pyruvate (a product of glycolysis). Treatment experiments on tumor-bearing mice reconfirmed that Gd-TCPP-NC can achieve excellent cancer therapeutic effects by combining SDT with starvation therapy through thiol-ene click reactions to kill tumors. Furthermore, the $r_{1}$ relaxation rate of Gd-TCPP-NC was as high as $51.7 \mathrm{~S}^{-1}\left(\mathrm{mmol} \mathrm{L}^{-1}\right)^{-1}$; further MRI application in tumor-bearing mice demonstrated it as an excellent $T_{1}$ contrast agent. This work suggests the potential of the thiol-ene click reaction in oncology combination therapy. Furthermore, this is a pioneering study that opens up future therapeutic integrations of antitumor therapies, the design of materials, and biological and medical areas.

\section{Received 1 August 2021; accepted 20 October 2021;} published online 10 December 2021

1 Son S, Kim JH, Wang X, et al. Multifunctional sonosensitizers in sonodynamic cancer therapy. Chem Soc Rev, 2020, 49: 3244-3261

2 Pan X, Wang W, Huang Z, et al. MOF-derived double-layer hollow nanoparticles with oxygen generation ability for multimodal imagingguided sonodynamic therapy. Angew Chem Int Ed, 2020, 59: 1355713561

3 Zhang D, Lin Z, Zheng Y, et al. Ultrasound-driven biomimetic nanosystem suppresses tumor growth and metastasis through sonodynamic therapy, CO therapy, and indoleamine 2,3-dioxygenase inhibition. ACS Nano, 2020, 14: 8985-8999

4 Yan P, Liu LH, Wang P. Sonodynamic therapy (SDT) for cancer treatment: Advanced sensitizers by ultrasound activation to injury tumor. ACS Appl Bio Mater, 2020, 3: 3456-3475
5 Xuan W, Xia Y, Li T, et al. Molecular self-assembly of bioorthogonal aptamer-prodrug conjugate micelles for hydrogen peroxide and $\mathrm{pH}$ independent cancer chemodynamic therapy. J Am Chem Soc, 2020, 142: 937-944

6 Wang X, Zhong X, Gong F, et al. Newly developed strategies for improving sonodynamic therapy. Mater Horiz, 2020, 7: 2028-2046

7 Cheng X, Xu HD, Ran HH, et al. Glutathione-depleting nanomedicines for synergistic cancer therapy. ACS Nano, 2021, 15: 8039-8068

8 Yang $\mathrm{F}, \mathrm{Li} \mathrm{M}$, Cui $\mathrm{H}$, et al. Altering the response of intracellular reactive oxygen to magnetic nanoparticles using ultrasound and microbubbles. Sci China Mater, 2015, 58: 467-480

9 Liu Z, Tang Z, Zhang D, et al. A novel GSH responsive poly(alphalipoic acid) nanocarrier bonding with the honokiol-DMXAA conjugate for combination therapy. Sci China Mater, 2019, 63: 307-315

10 Gong F, Cheng L, Yang N, et al. Ultrasmall oxygen-deficient bimetallic oxide $\mathrm{MnWO}_{X}$ nanoparticles for depletion of endogenous GSH and enhanced sonodynamic cancer therapy. Adv Mater, 2019, 31: 1900730

11 Chen Q, Feng L, Liu J, et al. Intelligent albumin- $\mathrm{MnO}_{2}$ nanoparticles as $\mathrm{pH}-/ \mathrm{H}_{2} \mathrm{O}_{2}$-responsive dissociable nanocarriers to modulate tumor hypoxia for effective combination therapy. Adv Mater, 2016, 28: 71297136

$12 \mathrm{Gu} \mathrm{T}$, Cheng L, Gong F, et al. Upconversion composite nanoparticles for tumor hypoxia modulation and enhanced near-infrared-triggered photodynamic therapy. ACS Appl Mater Interfaces, 2018, 10: 1549415503

13 Liu CP, Wu TH, Liu CY, et al. Self-supplying $\mathrm{O}_{2}$ through the catalaselike activity of gold nanoclusters for photodynamic therapy against hypoxic cancer cells. Small, 2017, 13: 1700278

14 Li M, Shao Y, Kim JH, et al. Unimolecular photodynamic $\mathrm{O}_{2}$-economizer to overcome hypoxia resistance in phototherapeutics. J Am Chem Soc, 2020, 142: 5380-5388

15 Liu HW, Hu XX, Li K, et al. A mitochondrial-targeted prodrug for NIR imaging guided and synergetic NIR photodynamic-chemo cancer therapy. Chem Sci, 2017, 8: 7689-7695

16 Kuang Y, Balakrishnan K, Gandhi V, et al. Hydrogen peroxide inducible DNA cross-linking agents: Targeted anticancer prodrugs. J Am Chem Soc, 2011, 133: 19278-19281

17 Yang Y, Chen M, Wang B, et al. NIR-II driven plasmon-enhanced catalysis for a timely supply of oxygen to overcome hypoxia-induced radiotherapy tolerance. Angew Chem, 2019, 131: 15213-15219

18 Qian X, Zheng Y, Chen Y. Micro/nanoparticle-augmented sonodynamic therapy (SDT): Breaking the depth shallow of photoactivation. Adv Mater, 2016, 28: 8097-8129

19 Liberti MV, Locasale JW. The warburg effect: How does it benefit cancer cells? Trends Biochem Sci, 2016, 41: 211-218

20 Wang YP, Zhou W, Wang J, et al. Arginine methylation of MDH1 by CARM1 inhibits glutamine metabolism and suppresses pancreatic cancer. Mol Cell, 2016, 64: 673-687

21 Li L, Liang Y, Kang L, et al. Transcriptional regulation of the Warburg effect in cancer by SIX1. Cancer Cell, 2018, 33: 368-385.e7

22 Yao Z, Zhang B, Liang T, et al. Promoting oxidative stress in cancer starvation therapy by site-specific startup of hyaluronic acid-enveloped dual-catalytic nanoreactors. ACS Appl Mater Interfaces, 2019, 11: 18995-19005

23 Zhu Y, Shi H, Li T, et al. A dual functional nanoreactor for synergistic starvation and photodynamic therapy. ACS Appl Mater Interfaces, 2020, 12: 18309-18318

24 Feng L, Xie R, Wang C, et al. Magnetic targeting, tumor microenvironment-responsive intelligent nanocatalysts for enhanced tumor ablation. ACS Nano, 2018, 12: 11000-11012

25 Yang B, Ding L, Chen Y, et al. Augmenting tumor-starvation therapy by cancer cell autophagy inhibition. Adv Sci, 2020, 7: 1902847

26 Yang X, Yang Y, Gao F, et al. Biomimetic hybrid nanozymes with selfsupplied $\mathrm{H}^{+}$and accelerated $\mathrm{O}_{2}$ generation for enhanced starvation and photodynamic therapy against hypoxic tumors. Nano Lett, 2019, 19: 4334-4342

27 Zhang L, Wang Z, Zhang Y, et al. Erythrocyte membrane cloaked metal-organic framework nanoparticle as biomimetic nanoreactor for starvation-activated colon cancer therapy. ACS Nano, 2018, 12: 10201- 


\section{1}

28 Deng Z, He Y, Younis MR, et al. Light-triggered plasmonic vesicles with enhanced catalytic activity of glucose oxidase for programmable photothermal/starvation therapy. Sci China Mater, 2020, 64: 1291-1301

29 Mahar R, Donabedian PL, Merritt ME. HDO production from $\left[{ }^{2} \mathrm{H} 7\right]$ glucose quantitatively identifies Warburg metabolism. Sci Rep, 2020, 10: 8885

30 Yun J, Mullarky E, Lu C, et al. Vitamin C selectively kills KRAS and BRAF mutant colorectal cancer cells by targeting GAPDH. Science, 2015, 350: 1391-1396

31 Li T, Liu M, Feng X, et al. Glyceraldehyde-3-phosphate dehydrogenase is activated by lysine 254 acetylation in response to glucose signal. J Biol Chem, 2014, 289: 3775-3785

32 Hoyle CE, Bowman CN. Thiol-ene click chemistry. Angew Chem Int Ed, 2010, 49: 1540-1573

33 Choi H, Kim M, Jang J, et al. Visible-light-induced cysteine-specific bioconjugation: Biocompatible thiol-ene click chemistry. Angew Chem Int Ed, 2020, 59: 22514-22522

34 Wang B, Durantini J, Nie J, et al. Heterogeneous photocatalytic click chemistry. J Am Chem Soc, 2016, 138: 13127-13130

35 Sticker D, Geczy R, Häfeli UO, et al. Thiol-ene based polymers as versatile materials for microfluidic devices for life sciences applications. ACS Appl Mater Interfaces, 2020, 12: 10080-10095

36 Kumari S, Malvi B, Ganai AK, et al. Functionalization of SBA-15 mesoporous materials using "thiol-ene click" michael addition reaction. J Phys Chem C, 2011, 115: 17774-17781

37 Baycan Koyuncu F, Davis AR, Carter KR. Emissive conjugated polymer networks with tunable band-gaps via thiol-ene click chemistry. Chem Mater, 2012, 24: 4410-4416

38 McCall JD, Anseth KS. Thiol-ene photopolymerizations provide a facile method to encapsulate proteins and maintain their bioactivity. Biomacromolecules, 2012, 13: 2410-2417

39 Hu Y, Tian Y, Cheng J, et al. Synthesis of eugenol-based polyols via thiol-ene click reaction and high-performance thermosetting polyurethane therefrom. ACS Sustain Chem Eng, 2020, 8: 4158-4166

40 Wang F, Zhang Y, Du Z, et al. Designed heterogeneous palladium catalysts for reversible light-controlled bioorthogonal catalysis in living cells. Nat Commun, 2018, 9: 1209

41 Huang P, Qian X, Chen Y, et al. Metalloporphyrin-encapsulated biodegradable nanosystems for highly efficient magnetic resonance imaging-guided sonodynamic cancer therapy. J Am Chem Soc, 2017, 139: $1275-1284$

42 Yang Y, Huo F, Yin C, et al. Thiol-chromene click chemistry: A coumarin-based derivative and its use as regenerable thiol probe and in bioimaging applications. Biosens Bioelectron, 2013, 47: 300-306

43 Zhao Y, Kuang Y, Liu M, et al. Synthesis of metal-organic framework nanosheets with high relaxation rate and singlet oxygen yield. Chem Mater, 2018, 30: 7511-7520

44 Nguyen Cao TG, Kang JH, You JY, et al. Safe and targeted sonodynamic cancer therapy using biocompatible exosome-based nanosonosensitizers. ACS Appl Mater Interfaces, 2021, 13: 25575-25588

45 Bai S, Yang N, Wang X, et al. Ultrasmall iron-doped titanium oxide nanodots for enhanced sonodynamic and chemodynamic cancer therapy. ACS Nano, 2020, 14: 15119-15130

46 Han X, Huang J, Jing X, et al. Oxygen-deficient black titania for synergistic/enhanced sonodynamic and photoinduced cancer therapy at near infrared-II biowindow. ACS Nano, 2018, 12: 4545-4555

47 Zhu P, Chen Y, Shi J. Nanoenzyme-augmented cancer sonodynamic therapy by catalytic tumor oxygenation. ACS Nano, 2018, 12: 37803795

48 Dinescu M, Perrone A, Caricato AP, et al. Boron carbon nitride films deposited by sequential pulses laser deposition. Appl Surf Sci, 1998, 127-129: 692-696

49 Ulrich S, Theel T, Schwan J, et al. Magnetron-sputtered superhard materials. Surf Coat Technol, 1997, 97: 45-59

50 Yue J, Cheng W, Zhang X, et al. Ternary BCN thin films deposited by reactive sputtering. Thin Solid Films, 2000, 375: 247-250

51 Maekawa $\mathrm{H}$, Ballano G, Toniolo $\mathrm{C}$, et al. Linear and two-dimensional infrared spectroscopic study of the amide I and II modes in fully ex- tended peptide chains. J Phys Chem B, 2011, 115: 5168-5182

52 Maekawa H, Ge NH. Comparative study of electrostatic models for the amide-I and -II modes: Linear and two-dimensional infrared spectra. J Phys Chem B, 2010, 114: 1434-1446

53 Zhao Y, Wang J, Cai X, et al. Metal-organic frameworks with enhanced photodynamic therapy: Synthesis, erythrocyte membrane camouflage, and aptamer-targeted aggregation. ACS Appl Mater Interfaces, 2020, 12: $23697-23706$

54 Kadish KM, E W, Zhan R, et al. Porphyrin-diones and porphyrintetraones: Reversible redox units being localized within the porphyrin macrocycle and their effect on tautomerism. J Am Chem Soc, 2007, 129: 6576-6588

55 Hussein BA, Shakeel Z, Turley AT, et al. Control of porphyrin planarity and aggregation by covalent capping: Bissilyloxy porphyrin silanes. Inorg Chem, 2020, 59: 13533-13541

56 Zhang R, Horner JH, Newcomb M. Laser flash photolysis generation and kinetic studies of porphyrin-manganese-oxo intermediates. Rate constants for oxidations effected by porphyrin- $\mathrm{Mn}^{\mathrm{V}}$-oxo species and apparent disproportionation equilibrium constants for porphyrin- $\mathrm{Mn}^{\mathrm{IV}}$ -oxo species. J Am Chem Soc, 2005, 127: 6573-6582

57 Gibilisco RG, Uranga JG, Santiago AN, et al. Atmospheric degradation of $\mathrm{CH}_{2}=\mathrm{C}\left(\mathrm{CH}_{3}\right) \mathrm{C}(\mathrm{O}) \mathrm{OCH}_{3}$ initiated by $\mathrm{OH}$ radicals: Mechanistic study and quantification of $\mathrm{CH}_{3} \mathrm{C}(\mathrm{O}) \mathrm{C}(\mathrm{O}) \mathrm{OCH}_{3}$ in $\mathrm{NO}_{x}$ free air. J Phys Chem A, 2015, 119: 8775-8783

58 Hayes JD, Dinkova-Kostova AT, Tew KD. Oxidative stress in cancer. Cancer Cell, 2020, 38: 167-197

59 Sun S, Oliveira BL, Jiménez-Osés G, et al. Radical-mediated thiol-ene strategy: Photoactivation of thiol-containing drugs in cancer cells. Angew Chem Int Ed, 2018, 57: 15832-15835

60 Oka M, Katsube D, Tsuji T, et al. Phototropin-inspired chemoselective synthesis of unsymmetrical disulfides: Aerobic oxidative heterocoupling of thiols using flavin photocatalysis. Org Lett, 2020, 22: 9244-9248

61 Stanisz GJ, Henkelman RM. Gd-DTPA relaxivity depends on macromolecular content. Magn Reson Med, 2000, 44: 665-667

62 Laurent S, Burtea C, Vander Elst L, et al. Synthesis and characterization of new low-molecular-weight lysine-conjugated Gd-DTPA contrast agents. Contrast Media Mol Imag, 2010, :

63 Li H, Meade TJ. Molecular magnetic resonance imaging with Gd(III)based contrast agents: Challenges and key advances. J Am Chem Soc, 2019, 141: 17025-17041

64 Caravan P. Strategies for increasing the sensitivity of gadolinium based MRI contrast agents. Chem Soc Rev, 2006, 35: 512-523

65 Wang L, Zhu X, Tang X, et al. A multiple gadolinium complex decorated fullerene as a highly sensitive $T_{1}$ contrast agent. Chem Commun, 2015, 51: 4390-4393

66 Zhu X, Lin $\mathrm{H}$, Wang L, et al. Activatable $T_{1}$ relaxivity recovery nanoconjugates for kinetic and sensitive analysis of matrix metalloprotease 2. ACS Appl Mater Interfaces, 2017, 9: 21688-21696

Acknowledgements This work was supported by the National Natural Science Foundation of China (52172096) and the Classification Development of the Capital Normal University (009-2155091).

Author contributions Jiang S, Zhang W, and Tian Y designed the experiments. Jiang $S$ performed the experiments and prepared the manuscript. He Q, Ye L, and Zhang W helped analyze some data on biology. Li C and Dang K helped analyze the data of the materials. Tian Y supervised the project and revised the manuscript.

Conflict of interest The authors declare that they have no conflict of interest.

Supplementary information Experimental details and supporting data are available in the online version of the paper. 


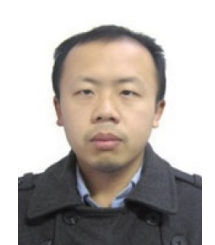

Sen Jiang is a postgraduate student in Prof. Tian's group and will receive his master degree from the Capital Normal University in 2022. His current research interest is synthesizing inorganic nanomaterials for tumor treatment.

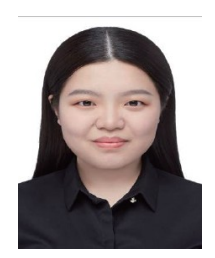

Qijia He received her bachelor degree of biology from the Capital Normal University. Now she is pursuing a master degree at the College of Life Sciences, Capital Normal University. Her research focuses on cancer biology.

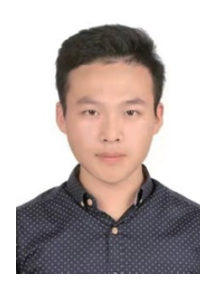

Kun Dang is a postgraduate student in Prof. Tian's group and will receive his master degree from the Capital Normal University in 2022. His current research interest is synthesizing inorganic nanomaterials for catalysis.

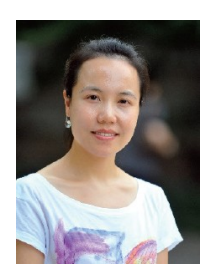

Weiwei Zhang received her bachelor degree of medicine from Peking University and $\mathrm{PhD}$ degree of biology from the National University of Singapore. She is now a professor and doctoral supervisor of the College of Life Sciences, Capital Normal University. Her research focuses on exploring the mechanisms underlying stem cell differentiation and tumorigenesis.

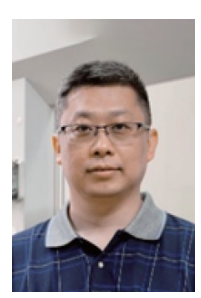

Yang Tian received his BS degree in 2002 and $\mathrm{PhD}$ degree in 2007 both from Shandong University. He then joined the Department of Chemistry, Capital Normal University in 2007, and visited the University of Notre Dame as a visiting scholar in 2013-2014. His current research focuses on the design and synthesis of novel inorganic nanomaterials for the applications of MRI imaging and cancer therapy, as well as oxygen evolution catalysts.

\section{通过金属有机框架材料产生巯基-烯点击反应促进声 动力-饥饿疗法治疗肿瘤}

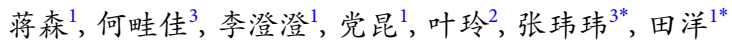

摘要 缺氧的肿瘤微环境(TME)限制了声动力疗法(SDT)杀灭癌细胞的 效果, 而且癌细胞中过量的谷胱甘肽 $(G S H)$ 进一步加剧了这种疗效限 制. 同时, 癌症饥饿疗法作为一种有前途的临床治疗手段已得到越来越 多的认可, 但基于葡萄糖氧化酶(GOx)的饥饿疗法的疗效也因缺氧问题 而受到限制. 甘油醛-3-磷酸脱氢酶 (GAPDH) 是一种关键的糖酵解酶, 可作为缺氧情况下饥饿疗法的靶点. 本文提出利用二维金属有机框架 (MOF) 实现颈基-烯点击反应, 达到对肿瘤进行SDT和饥饿疗法联合治 疗的目的. 实验结果表明, 因所制备的MOF材料和癌细胞中硫醇物质 发生颈基-烯点击反应，该MOF可以在TME中无氧消耗GSH和GAPDH. 进一步的体外和体内实验表明, 所制备的MOF材料可以有效地杀死癌 细胞. 这项研究有望为統基-烯点击反应在SDT疗法和癌症饥饿疗法的 应用中开辟一条前景广阔的道路. 